\title{
An Energy Measurement Method of Electric Vehicle Based on FFT and Wavelet Transform
}

\author{
Zhengqi Tian, Jian Liu, Qing Xu, Shunran Qi, and Guangwei Chen
}

\begin{abstract}
Electric vehicle charging process of nonlinear load caused by harmonic components affects the accuracy of energy metering. The energy metering algorithm of electric vehicle is constructed by combining Fast Fourier Transformation algorithm (FFT) with the wavelet time scale transform method, then FFT can analyze steady-state harmonic and wavelet algorithm can deal with the singular signals effectively. According to the approximation parameters and local characteristic parameters of wavelet analysis, a high resolution harmonic analysis model is established. Then, the wavelet decomposition number of the signal and reconstruction are determined by fundamental signal frequency and sampling frequency, steady-state and non-steady-state harmonics are identified and separated, and the harmonic energy metering is achieved. Simulation demonstrates that FFT and wavelet transform of the electric vehicle energy measurement method can effectively extract the order of the harmonic signal, track and observe the band within the harmonic changes, and improve the measurement accuracy of the electric vehicle charging electric power.
\end{abstract}

Index Terms-Electric vehicles charger, Harmonic wave, Electric power energy measurement, Fourier transform algorithm, Wavelet transform.

\section{INTRODUCTION}

Electric vehicles have outstanding advantages of energy-saving, environmental protection, and are vigorously promoting to be used all the world. However, Electric vehicle battery charging is a nonlinear capacitive load, the load distribution is rather random, and load power factor is low [1], [2]. In addition, the complexity of the charging process will produce shock load, nonlinear load and transient harmonics [3], and bring harmonic pollution and electric energy measurement error. To ensure accurate and reliable measurement, electric vehicle charging station energy metering device must have harmonic measurement, monitoring, DC energy metering and wide load measurement function. It is crucial for energy meter in electric vehicle charging station to achieve accurate measurement under complex load conditions. In practice, the harmonic signal induced by electric vehicle charging process changes in real-time, and complexity of harmonics alters constantly [4], [5], such as instantaneous mutation signals, white noise interference and so on. FFT algorithm

Manuscript received October 9, 2017; revised November 25, 2017.

Zhengqi Tian, Jian Liu, and Qing Xu are with Jiangsu Electric Power Company Research Institute, China (e-mail: wlhseu@163.com).

Shunran Qi is with School of Instrument Science and Engineering, Southeast University, China.

Guangwei Chen is with Purdue University, China. can neither approximate the measured signal in the time domain and frequency domain, nor accurately obtain the harmonic signal amplitude, frequency and phase. FFT algorithm has a great limitation for harmonic detection, and is difficult to complete the accurate measurement of electric vehicle charging power.

Based on the FFT, short-time Fourier transform (STFT) technique uses a center-symmetric sliding window function to intercept the observed signals. STFT realizes the time-frequency localization analysis of the signal by sliding the window function in the time domain[6], to enhance the analysis ability of the non-stationary signal, and also reduce the error caused by the leakage of the spectrum. However, STFT transform still has shortcomings in time-frequency resolution [7], [8], and the time-domain waveform and spectrum of the signal can not obtain high resolution at the same time, and its observation window is hardened and the local observation characteristic is not detailed enough. STFT algorithm is still difficult to complete the accurate measurement of electric vehicle charging power.

Achieving the fine signal analysis of electric power from the time domain and frequency domain is the core step for harmonic detection. This paper presents an improved non-linear harmonic energy measurement method for electric vehicle charging, the steady-state and non-steady-state harmonics in non-linear load electric vehicle charging operation can be separated, the multi-scale refinement analysis of the nonlinear harmonic signal of electric power is carried out by using time scale and translation operations, then, both the time-domain waveform and the spectrum of the signal can be achieved simultaneously with high resolution. Energy measurement method of electric vehicle based on FFT and Wavelet transform presented here can solve the problem that the FFT algorithm is not sufficient in the non-linear load electric power measurement, and improve the accuracy of the charging electric energy measurement of the electric vehicle.

\section{LOAD CHARACTERISTICS OF ELECTRIC VEHICLES}

Electric vehicles have two kinds of charging mode, including conventional charging and fast charging. During charging process, the distribution of the charge load is very random in both time and space [9]. Conventional charging mode adopts a small charging current with constant voltage or constant current, charging current is about $15 \mathrm{~A}$, the charging time is generally 5 to 8 hours [10]. Fast charge mode adopts $150 \sim 400 \mathrm{~A}$ charging current, the battery reaches or closes to the full state within $20 \mathrm{~min}$ to $2 \mathrm{~h}$, and the battery state of charge (SOC) can be improved in a very 
short period.

Due to load nonlinearity of electric vehicle charging, the power supplied to the load is a sinusoidal voltage, while the current is a non-sinusoidal waveform composed of the fundamental and harmonics [11]. Non-linear loads cause harmonics, resulting in an increase of harmonic components in the power supply. Power supply voltage distortion decreases the system power factor, affects power quality, then affects the accuracy of energy measurement. Fig. 1 is the equivalent circuit model of the electric vehicle charging load,

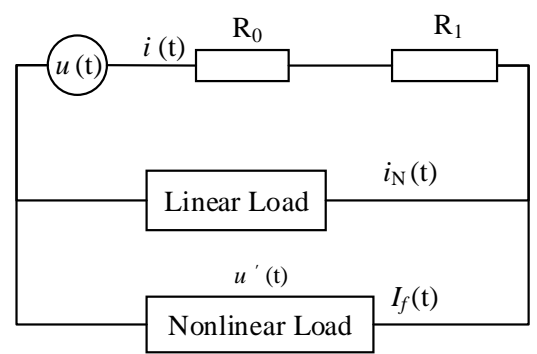

Fig. 1. Equivalent circuit model of the electric vehicle charging load.

$\mathrm{R}_{0}$ is the internal resistance of the power supply, $\mathrm{R} 1$ is the line resistance. The power supply $u(t)$ is a standard sinusoidal voltage source, the total load is divided into linear load and non-linear load, the total non-linear load current $i^{\prime}(t)$ is divided into linear load fundamental current $i_{N}(t)$ and non-linear load harmonic current $i_{f}(t)$, the total non-linear load voltage $u(t)$ is divided into linear load fundamental current $u_{N}(t)$ and non-linear load harmonic current $u_{f}(t)$.

The electric power $\mathrm{P}$ delivered by the power supply is obtained by

$$
\begin{gathered}
P=\frac{1}{T} \int_{0}^{T} u(t) i(t) d t=\frac{1}{T} \int_{0}^{T}(R+R 1) i^{2}(t) d t+\frac{1}{T} \int_{0}^{T} u^{\prime}(t) i(t) d t \\
i(t)=i_{N}(t)+i_{f}(t) \\
u^{\prime}(t)=u_{N}(t)+u_{f}(t)
\end{gathered}
$$

Then the electric power $P$ is obtained by

$$
\begin{aligned}
& P=\frac{1}{T} \int_{0}^{T}(R+R 1) i_{N}^{2}(t) d t+\frac{1}{T} \int_{0}^{T}(R+R 1) i_{f}^{2}(t) d t+\frac{1}{T} \int_{0}^{T} u_{N}(t) i_{N}(t) d t \\
& +\frac{1}{T} \int_{0}^{T} u_{f}(t) i_{f}(t) d t+\frac{1}{T} \int_{0}^{T} u_{N}(t) i_{f}(t) d t+\frac{1}{T} \int_{0}^{T} u_{f}(t) i_{N}(t) d t \\
& +\frac{2}{T} \int_{0}^{T}(R+R 1) i_{N}(t) i_{f}(t) d t
\end{aligned}
$$

where, $\frac{1}{T} \int_{0}^{T}(R+R 1) i_{N}(t) i_{f}(t) d t=0, \quad \int_{0}^{T} u_{N}(t) i_{f}(t) d t=0$,

$$
\begin{array}{ll}
\frac{1}{T} \int_{0}^{T} u_{f}(t) i_{N}(t) d t=0 \quad, & P_{1}=\frac{1}{T} \int_{0}^{T}(R+R 1) i_{N}{ }^{2}(t) d t, \\
P_{2}=\frac{1}{T} \int_{0}^{T} u_{N}(t) i_{N}(t) d t \quad, & P_{3}=\frac{1}{T} \int_{0}^{T}(R+R 1) i_{f}{ }^{2}(t) d t, \\
P_{4}=\frac{1}{T} \int_{0}^{T} u_{f}(t) i_{f}(t) d t 。 &
\end{array}
$$

where $P_{1}$ is the fundamental power of source internal resistance and the line resistance, $P_{2}$ is the fundamental power of linear load, $P_{3}$ represents the harmonic power of source internal resistance and electric line, $P_{4}$ represents the harmonic power of non-linear load

\section{Characteristic OF Energy Metering Error}

Electronic energy meter on the load measurement is the fundamental and harmonic power of the integrated power, that is, the whole energy mode. This measurement method adds fundamental active power and harmonic load active energy consumption algebraically [12]. For the harmonic source users, the harmonic energy is opposite to the fundamental energy consumed by users. That is the fundamental energy consumed by users minus the harmonic energy they emit. Electronic energy meter is classified into analog multiplier-type energy meter and digital energy meter, and the type of digital energy meter is commonly used for electric vehicle charging.

Digital energy meter adopts discrete harmonic integral algorithm. The total active electric power is the sum of the fundamental-wave power, the power of the same order harmonic current and voltage [13], the power of the different order harmonic current and voltage. For the whole cycle of integral calculation, the power generated among different order harmonics in a cycle average power is zero, then integral calculation cannot separate the harmonic power and its plus or minus.FFT algorithm is commonly used during discrete integral process. FFT decomposes the periodic signal into a superposition of different frequency components according to the Fourier series, then the electric power-related parameters are calculated by using the separated signals.

The voltage and current signals of electric vehicle charging system contain multiple harmonic components, and the fundamental frequency will drift [14]. FFT algorithm uses the spectral analysis to detect harmonic components, with advantages of fast response, high precision, real-time. However, due to the signal truncation, non-synchronous sampling, and non-integer period sampling, FFT algorithm exists the deficient of spectral leakage. By modifying the FFT algorithm, spectral leakage is reduced, and the accuracy of harmonic detection is improved. A suitable window function can be used to restrain the spectral leakage caused by signal truncation error, while the amount of computation is increased. Moreover, interpolation algorithm is used to correct the FFT result (amplitude and phase) to reduce the deviation of peak observation points caused by the fence effect.

During electric vehicle charging process, the harmonic signal changes in real time. As a global method of mathematical change, FFT isn't capable of the localized analysis, and not suitable for analyzing non-stationary harmonic signals. Wavelet analysis performs multi-scale refinement analysis of a function or signal by using the expansion, contraction and other computing functions, then extracts the harmonic information effectively with time and frequency domain local transform. Wavelet analysis can 
compensate for the shortcomings of FFT and SFFT algorithms, and integrate with FFT algorithm, which is suitable for the complicated running process of the electric energy metering system.

\section{Energy MEASUREMEnt Algorithm Based on FFT AND WAVELET FUSION}

From the perspective of time domain or frequency domain, it is a core step to achieve the fine signal analysis for harmonic detection. FFT algorithm has rather excellent frequency resolution and measurement accuracy in the steady-state harmonic detection [15], [16]. The shortcoming of FFT is that it hasn't a localized analysis ability, and not suitable for analyzing non-stationary signals as a global mathematical change.

Wavelet transform has the advantages of time domain and frequency domain localization, direction selectivity and variable time-frequency resolution based on time-scale analysis. Wavelet transform overcomes the shortcoming that the FFT algorithm smooth the local signals in the whole domain, and can reflect the details of the signals. Wavelet transform can adjust the width of the time window and the frequency window [17]. The sampling density is automatically adjusted to handle the mutated signal according to the frequency components of the signal, then, sudden changes in the signal and time-varying tracking can be identified. Wavelet transform is suitable for fluctuating harmonics, rapid changes of harmonics, mutations and non-stationary signal analysis, and applicable for harmonic measurement in the charging process of electric vehicles. Wavelet transform makes up the shortcomings of Fourier transform in the unsteady signal processing [18], [19]. However, it cannot achieve accurate measurement of harmonic spectrum information in steady-state harmonic processing. The combination of wavelet transform and Fourier transform can realize the seamless measurement of electric energy in the whole process of electric vehicle charging.

For the power harmonic signal $f(\mathrm{t})$, it is decomposed into low-frequency characteristics of the approximation signal and high-frequency characteristics of the details of the signal. The $f(\mathrm{t})$ decomposed with $\mathrm{j}$ scale is obtained by

$$
f(\mathrm{t})=\sum_{k} c_{j, k} 2^{-j / 2} \varphi\left(2^{-j} t-k\right)+\sum_{k} d_{j, k} 2^{-j / 2} \psi\left(2^{-j} t-k\right)
$$

where $\varphi(*)$ is scale space function, $\psi(*)$ is wavelet mother function, $\sum_{k} c_{j, k} 2^{-j / 2} \varphi\left(2^{-j} t-k\right)$ is scale projection used for decomposition of the low frequency part of the signal.

The multi-resolution method in wavelet transform divides the frequency band of each layer, then, the approximation parameter and local characteristic parameter are obtained by

$$
c_{j+1, k}(\mathrm{k})=\sqrt{2} \sum_{m} h(\mathrm{~m}-2 \mathrm{k}) c_{j, m}
$$

$$
d_{j+1, k}(\mathrm{k})=\sqrt{2} \sum_{m} g(\mathrm{~m}-2 \mathrm{k}) c_{j, m}
$$

where $c_{j, k}$ is the approximation wavelet parameter with $\mathrm{j}$ scale, $d_{j, k}$ is the local characteristic wavelet parameter with $\mathrm{j}$ pace, $\mathrm{k}$ is the harmonic number, $\psi_{a, \tau}(*)$ is the wavelet basis function $\psi_{a, \tau}(*)=\frac{1}{\sqrt{a}} \psi\left(\frac{a-\tau}{a}\right), \quad a$ is the scale factor, $\tau$ is the translation factor, $m$ is power series with scale discretization, $h\left(^{*}\right)$ is the scale space filter function, $h\left(^{*}\right)$ expresses the low-pass signal's frequency characteristics, $g(*)$ is wavelet space filter function, $g(*)$ expresses the high-pass signal's frequency characteristics.

Separation of different number harmonic can be achieved by using Eq.5 and Eq.6. After decomposition, each band contains different harmonic components, then, each frequency band is reconstructed, and the harmonics are obtained. Reconstruction formula is given by

$$
c_{j-1, \mathrm{~m}}=\sqrt{2} \sum_{k} c_{j, \mathrm{k}} h(\mathrm{~m}-2 \mathrm{k})+\sqrt{2} \sum_{k} d_{j, \mathrm{k}} \mathrm{g}(\mathrm{m}-2 \mathrm{k})
$$

The reconstructed frequency bands contain different harmonic components, and the unsteady harmonic components are separated from steady - state harmonic components. A reasonable division of the number of layers can ensure that the harmonics of the same frequency band do not produce aliasing. According to the frequency of the fundamental signal and the sampling frequency, the decomposition of the number of layers are determined. The number of decomposition layers is obtained by

$$
p=\log _{2}\left[f_{s} /\left(f_{0} \sqrt{8}\right)\right]+0.5
$$

where $f_{s}$ is sampling frequency, $f_{0}$ is fundamental frequency.

The voltage and current signals of the nonlinear load harmonics can be obtained by

$$
\begin{aligned}
& u(\mathrm{t})=\sum_{i=0}^{2^{j}-1} \sum_{k=0}^{2^{N-j}-1} d_{u j}^{(2 i)}(\mathrm{k}) \psi_{j, k}^{2 i}(\mathrm{t})+\sum_{i=0}^{2^{j}-1} \sum_{k=0}^{2^{N-j}-1} d_{u j}^{(2 i+1)}(\mathrm{k}) \psi_{j, k}^{(2 i+1)}(\mathrm{t}) \\
& =\sum_{k=0}^{2^{N-j}-1} d_{u j}^{(0)}(\mathrm{k}) \varphi_{j, k}(\mathrm{t})+\sum_{i=0}^{2^{j}-1} \sum_{k=0}^{2^{N-j}-1} d_{u j}^{(2 i)}(\mathrm{k}) \psi_{j, k}^{(i)}(\mathrm{t}) \\
& i(\mathrm{t})=\sum_{i=0}^{2^{j}-12^{N-j}} \sum_{k=0}^{(2 i)} d_{i j}^{(\mathrm{k})} \psi_{j, k}^{2 i}(\mathrm{t})+\sum_{i=0}^{2^{j}-12^{N-j}-1} \sum_{k=0}^{(2 i+1)}(\mathrm{k}) \psi_{j, k}^{(2 i+1)}(\mathrm{t}) \\
& =\sum_{k=0}^{2^{N-j}-1} d_{i j}^{(0)}(\mathrm{k}) \varphi_{j, k}(\mathrm{t})+\sum_{i=0}^{2^{j}-1} \sum_{k=0}^{2^{N-j}-1} d_{i j}^{(2 i)}(\mathrm{k}) \psi_{j, k}^{(i)}(\mathrm{t})
\end{aligned}
$$

where $j$ is the number of decomposed layers, $d_{u j}^{(0)}(\mathrm{k})$ is the coefficient of scale functions in reconstructed voltage signals, $d_{i j}^{(0)}(\mathrm{k})$ is the coefficient of scale functions in 
reconstructed current signals, $d_{u j}^{(i)}(\mathrm{k})$ is wavelet packet transform coefficient in reconstructed voltage signals, $d_{i j}^{(i)}(\mathrm{k})$ is wavelet packet transform coefficient in reconstructed current signals.

In the electric energy meter, electric power is achieved by multiplying the voltage and current, and accumulates in the time, and the power consumption is obtained by accumulating the power in time. Harmonic active power is obtained by

$$
\begin{gathered}
P=\frac{1}{T} \int_{0}^{T} u(\mathrm{t}) \cdot \mathrm{i}(\mathrm{t}) \mathrm{dt}=\frac{1}{2^{N}} \sum_{i=0}^{2^{j}-1} \sum_{k=0}^{2^{N-j}-1} d_{u j}^{(i)}(\mathrm{k}) d_{i j}^{(i)}(\mathrm{k})=\sum_{i=0}^{2^{j}-1} P_{j}^{(i)} \\
P_{j}^{(i)}=\frac{1}{2^{N}} \sum_{k=0}^{2^{N-j}-1}\left[d_{u j}^{(i)}(\mathrm{k}) d_{i j}^{(i)}(\mathrm{k})\right]
\end{gathered}
$$

where $P_{j}^{(i)}$ is the in-band harmonic signal active power at the $i$-th node with $\mathrm{j}$ decomposition scale.

\section{Simulation AnAlysis}

Several typical nonlinear load harmonic models of the electric vehicle charging process are simulated, then, energy measurement error characteristics with using FFT and wavelet are demonstrated respectively. The measurement signal contains the fundamental, third harmonic, intermittent and non-steady-state harmonics. Sampling frequency is $6400 \mathrm{~Hz}$, fundamental frequency is $50 \mathrm{~Hz}$.

Condition 1 . The voltage $\mathrm{u}(\mathrm{t})$ and currenti( $\mathrm{t})$ signals are given by

$$
\begin{aligned}
u(t)= & 100 \sin \sin (2 \pi \times 50 t)+20 \sin \sin (2 \pi \times 150 t) \\
& +5 \sin \sin (2 \pi \times 325 t) \\
i(t)= & 100 \sin \sin (2 \pi \times 50 t)+12.5 \sin \sin (2 \pi \times 150 t) \\
+ & 4 \sin \sin (2 \pi \times 325 t)
\end{aligned}
$$

In $4 T \leq t \leq 8 T$ time, the non-steady-state signals are added to the voltage and current signals, where $T$ is fundamental period.

$$
\begin{gathered}
u(t)=10 e^{-40 t} \sin \sin (2 \pi \times 550 t) \\
i(t)=12 e^{-40 t} \sin \sin (2 \pi \times 550 t)
\end{gathered}
$$

Fig. 2 shows the original voltage signal waveforms, including fundamental wave, third harmonic, intermittent wave, non-steady-state harmonic and integrated signal. Wavelet algorithm analysis of the voltage signal reconstructed waveform is shown in Fig. 3. Wavelet algorithm analysis of the current signal reconstructed waveform is shown in Fig. 4. Wavelet transform and FFT algorithm are used to analyze the above-mentioned signals, and the energy metering data and error analysis of the 16 fundamental wave periods are shown in Table I.

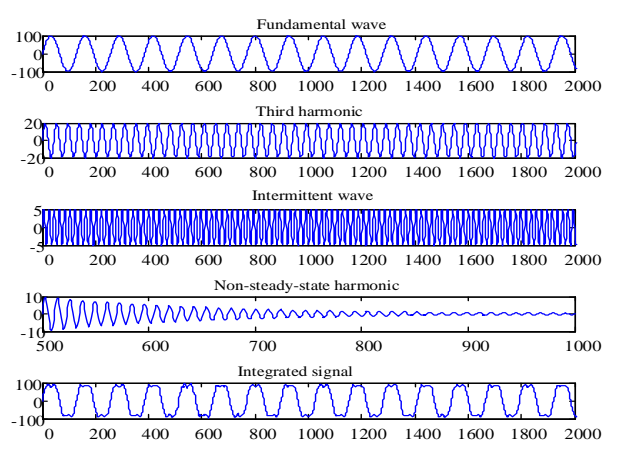

Fig. 2. Original voltage signal waveforms.



Fig. 3. Reconstructed waveforms of voltage signal.
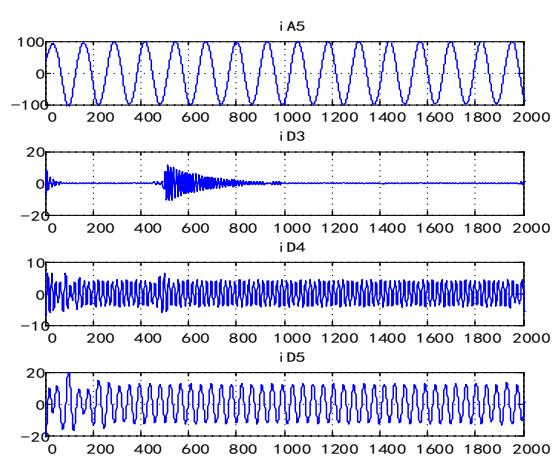

Fig. 4. Reconstructed waveforms of current signal.

TABLE I: ENERGY METERING DATA AND ERROR ANALYSIS

\begin{tabular}{|c|c|c|c|c|c|}
\hline \multirow{2}{*}{ Frequency/Hz } & $\begin{array}{c}\text { Theoretical value of power } \\
\text { energy /J }\end{array}$ & \multicolumn{2}{|c|}{ Value of power energy by wavelet transform } & \multicolumn{2}{|c|}{ Value of power energy by FFT } \\
\cline { 2 - 5 } & 1600 & 1600.4039 & $2.5243682 \mathrm{e}-4$ & 1599.654613 \\
\hline 50 & 40 & 39.88965 & $2.75873450 \mathrm{e}-3$ & 39.892497 & $2.68757326 \mathrm{e}-3$ \\
\hline 150 & 3.2 & 3.4935 & $9.17192251 \mathrm{e}-2$ & 3.238558765 \\
\hline 325 & 0.7499 & 0.8565 & $1.42182233 \mathrm{e}-1$ & 0.107133617 \\
\hline 550 & & & $8.57136039 \mathrm{e}-1$ \\
\hline
\end{tabular}


Condition 2. The voltage $u(t)$ and current $i(t)$ signals are given by

$$
\begin{aligned}
u(t)= & 100 \sin \sin (2 \pi \times 50 t)+20 \sin \sin (2 \pi \times 150 t) \\
& +5 \sin \sin (2 \pi \times 325 t) \\
i(t)= & 100 \sin \sin (2 \pi \times 50 t)+12.5 \sin \sin (2 \pi \times 150 t) \\
& +4 \sin \sin (2 \pi \times 325 t)
\end{aligned}
$$

In $4 T \leq t \leq 8 T$ time, the non-steady-state signals are added to the voltage and current signals, where $T$ is fundamental period.

$$
\begin{aligned}
& u(t)=4 e^{-40 t} \sin \sin (2 \pi \times 1050 t) \\
& i(t)=5 e^{-40 t} \sin \sin (2 \pi \times 1050 t)
\end{aligned}
$$

\begin{tabular}{|c|c|c|c|c|c|}
\hline \multirow{2}{*}{ Frequency/Hz } & \multirow{2}{*}{$\begin{array}{c}\text { Theoretical value of } \\
\text { power energy /J }\end{array}$} & \multicolumn{2}{|c|}{ Value of power energy by wavelet transform } & \multicolumn{2}{|c|}{ Value of power energy by FFT } \\
\hline & & Actual value /J & Relative error & Actual value /J & Relative error \\
\hline 50 & 1600 & 1600.6779 & $4.23659093 \mathrm{e}-4$ & 1599.9305 & $4.34657625 \mathrm{e}-5$ \\
\hline 150 & 40 & 39.9762 & $5.93957009 \mathrm{e}-4$ & 39.9796 & $5.09353408 \mathrm{e}-4$ \\
\hline 325 & 3.2 & 3.4464 & $7.70133715 \mathrm{e}-2$ & 3.2056 & $1.73726578 \mathrm{e}-3$ \\
\hline 1050 & 0.1250 & 0.1296 & $3.65144862 \mathrm{e}-2$ & 0.01785 & $8.57182035 \mathrm{e}-1$ \\
\hline
\end{tabular}

Wavelet transform and FFT algorithm are used to analyze the above-mentioned signals, and the energy metering data and error analysis of the 16 fundamental wave periods are shown in Table II.

TABLE II: ENERGY METERING DATA AND ERROR ANALYSIS

Condition 3. The voltage $u(t)$ and current $i(t)$ signals are given by

$$
\begin{aligned}
u(t)= & 100 \sin \sin (2 \pi \times 50 t)+20 \sin \sin (2 \pi \times 150 t) \\
& +5 \sin \sin (2 \pi \times 550 t) \\
i(t)= & 100 \sin \sin (2 \pi \times 50 t)+12.5 \sin \sin (2 \pi \times 150 t) \\
+ & 4 \sin \sin (2 \pi \times 550 t)
\end{aligned}
$$

In $4 T \leq t \leq 8 T$ time, the non-steady-state signals are added to the voltage and current signals, where $T$ is fundamental period.

$$
\begin{aligned}
& u(t)=24 e^{-40 t} \sin \sin (2 \pi \times 1050 t) \\
& i(t)=22 e^{-40 t} \sin \sin (2 \pi \times 1050 t)
\end{aligned}
$$

With Condition 3, the energy metering data and error analysis of the 16 fundamental wave periods are shown in Table III.

TABLE III: ENERGY METERING DATA AND ERROR ANALYSIS

\begin{tabular}{|c|c|c|c|c|c|}
\hline \multirow{2}{*}{ Frequency/Hz } & \multirow{2}{*}{$\begin{array}{c}\text { Theoretical value of } \\
\text { power energy /J }\end{array}$} & \multicolumn{2}{|c|}{ Value of power energy by wavelet transform } & \multicolumn{3}{|c|}{ Value of power energy by FFT } \\
\cline { 3 - 6 } & & Actual value /J & Relative error & Actual value /J & Relative error \\
\hline 50 & 1600 & 1600.2982 & $1.86342296 \mathrm{e}-4$ & 1599.6446 & $2.22128541 \mathrm{e}-4$ \\
\hline 150 & 40 & 39.9860 & $3.51185285 \mathrm{e}-4$ & 39.8995 & $2.51144564 \mathrm{e}-3$ \\
\hline 550 & 3.2 & 0.2978 & $9.06941401 \mathrm{e}-1$ & 3.1748 & $7.86968970 \mathrm{e}-3$ \\
\hline 1050 & 3.2999 & 3.2413 & $1.77492863 \mathrm{e}-2$ & 0.4713 & $8.57182035 \mathrm{e}-1$ \\
\hline
\end{tabular}

Simulation results of three models demonstrate that FFT is more accurate than the wavelet for steady-state harmonic measurements, however, it is difficult for FFT to discern the frequency of the intermittent waves, which causes spectral leakage and it is very inaccurate for FFT in non-steady-state harmonic measurements. Moreover, wavelet transform can effectively extract the order of the harmonic signal, track and observe the changes in frequency band of the harmonics, then, achieve accurate measurement of non-stationary harmonic electric energy. Combining FFT algorithm with wavelet transform algorithm is an effective method to solve the electric vehicle harmonic energy measurement problem caused by nonlinear load.

\section{Conclusions}

The non-linear loads in the charging process of electric vehicles cause the voltage and current harmonic signals to be dynamically time-varying. FFT algorithm cannot approximate the measured signal in the time domain and frequency domain at any time, then, cannot accurately obtain the amplitude, frequency and phase of each harmonic signal, either. It is difficult for FFT algorithm to achieve accurate measurement of electric vehicle charging power. According to the dynamic time-varying and non-linear characteristic of electric vehicle charging load, a wavelet transform algorithm is proposed. The signals of different frequency magnitudes are carefully observed, and the non-stationary components in 
the signal can be distinguished effectively. The entropy value of wavelet algorithm can locate the time of disturbance and extract the relevant information of disturbance signal. Accurate energy measurement of the electric vehicle steady-state, non-steady-state fundamental power and the harmonic is realized, by combining with FFT algorithm to analyze steady-state harmonic and wavelet algorithm in singular signal processing. Electric vehicle charging station watt-hour meter with full-wave energy meter can effectively suppress the impact of harmonics on energy measurement error.

\section{REFERENCES}

[1] J. T. Yang, J. Le et al., "Analysis of Measurement Error for Electric Energy Metering System under harmonic", Automation of Electric Power, vol. 39, no. 13, pp. 144-150, 2015.

[2] S. K. Jain and S. N. Singh, "Exact model order ESPRIT technique for harmonics and inter harmonics estimation," IEEE Trans. Instrum Meas., vol. 61, pp. 1915-1923, 2012.

[3] H. L. Cai and K. Xiao, "Application of wavelet packet improved reconstruction algorithm to energy measurement of non-linear loads," Journal of System Simulation, vol. 27, pp. 2822-2828, 2015.

[4] M. Bertocco, G. Frigo, C. Narduzzi, and F. Tramarin, "Resolution enhancement by compressive sensing in power quality and phasor measurement," IEEE Trans. Instrum. Meas., vol. 63, pp. 2358-2367, 2014.

[5] X. H. Han, S. M. Shen, T. Xiao, et al, "Distortion signal energy metering based on morphological wavelet," Electrical Measurement Instrumentation, vol. 53, no. 10, pp. 44-51, 2016.

[6] R. K. Vasudevan, M. B. Okatan, I. Rajapaksa et al., "Higher order harmonic detection for exploring nonlinear interactions with nanoscale resolution," Scientific Reports, vol. 3, pp. 2677-2677, 2013.

[7] C. He and Q. Shu, "Separation and analyzing of harmonics and inter-harmonics based on single-channel independent component analysis," Int. Trans. Electr. Energy Syst., vol. 25, pp. 169-179, 2015.

[8] X. G. Zhu, Y. Qin, X. F. Su et al., "Impact of electric vehicle charger on electric energy metering," Automation of Electric Power, vol. 35, no. 6, pp. 52-57, 2015.

[9] Q. Zhang, W. J. Han, J. H. Yu et al., "Simulation model of electric vehicle charging station and the harmonic analysis on power grid," Transactions of China Electrotechnical Society, vol. 27, no. 2, pp. 159-164, 2012.

[10] W. Zhao, H. Y. Peng, W. M. Sun et al., "An electricity metering scheme based on quantitative metering error," Automation of Electric Power, vol. 539, no. 12, pp. 121-12, 2015.

[11] Y. M. Ni and X. M. Zhang, "Research on a new method of rational electric energy measurement under the distorted signals condition," Automation of Electric Power, vol. 32, no. 17, pp. 88-91, 2008.

[12] N. Li and M. Huang, "Analysis on harmonics caused by connecting different types of electric vehicle chargers with power network," Power System Technology, vol. 35, no. 1, pp. 170-174, 2011.

[13] S. B. Yang, M. L. Wu, J. C. Jiang et al., "An approach for load modeling of electric vehicle charging station," Power System Technology, vol. 37, no. 5, pp. 1190-1195, 2013.
[14] N. K. Clement, E. Haesen, and J. Driesen, "The impact of charging plug-in hybrid electric vehicles on a residential distribution grid," IEEE Transactions on Power Systems, vol. 25, no. 1, pp. 371-380, 2010.

[15] J. Wu, X. Wang, X. Sun, and Y. Liu, "Pure harmonics extracting from time-varying power signal based on improved empirical mode decomposition," Measurement, vol. 49, pp. 216-225, 2014.

[16] J. Justine, O. Fiona, and N. Tim, "Electric vehicles in Australia's national electricity market: Energy market and policy implications," The Electricity Journal, vol. 25, no. 2, pp. 63-87, 2012.

[17] J. L. Guo and F. S. Wen, "Impact of electric vehicle charging on power system and relevant countermeasures," Electric Power Automation Equipment, vol. 35, no. 6, pp. 1-9, 2015.

[18] Y. C. Liu, X. Wang, Y. D. Liu et al., "Resolution-enhanced harmonic and interharmonic measurement for power quality analysis in cyber-physical energy system," Sensors, vol. 16, no. 7, pp. 1-21, 2016.

[19] J. M. Guerrero, P. C. Loh, T. L. Lee, and M. Chandorkar, "Advanced control architectures for intelligent microgrids-part II: Power quality, energy storage, and AC/DC microgrids," IEEE Trans. Ind. Electron., s vol. 60 , pp. 1263-1270, 2013.



Zhengqi Tian was born in Jiangsu, China in 1987. $\mathrm{He}$ is a senior engineer in Jiangsu Electric Power Company Research Institute, China. His major research interests include filtering techniques in control system, and measurement in electric power system.

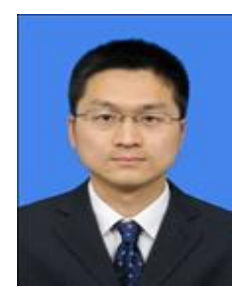

Jian Liu was born in Henan, China in 1981. He is a senior engineer in Jiangsu Electric Power Company Research Institute, China. His major research interests include filtering techniques in control system, and measurement in electric power system.

Qing Xu was born in Jiangsu, China in 1973. She is a senior engineer in Jiangsu Electric Power Company Research Institute, China. Her major research interests include filtering techniques in control system, and measurement in electric power system.

Shunran Qi was born in Henan, China in 1993. He is a postgraduate student in the School of Instrument Science and Engineering, Southeast University. His major research interests include filtering techniques in control system, and measurement in electric power system.

Guangwei Chen was born in Henan, China in 1998. He is a postgraduate student in Purdue University. His major research interests include filtering techniques in control system, and measurement in electric power system. 